\title{
VARIABLE STRUCTURE CONTROL WITH CHATTERING REDUCTION OF A GENERALIZED T-S MODEL
}

\author{
Basil M. Al-Hadithi, Agustín Jiménez, and Fernando Matía
}

\begin{abstract}
In this paper, a fuzzy logic controller (FLC) based variable structure control (VSC) is presented. The main objective is to obtain an improved performance of highly non-linear unstable systems. New functions for chattering reduction and error convergence without sacrificing invariant properties are proposed. The main feature of the proposed method is that the switching function is added as an additional fuzzy variable and will be introduced in the premise part of the fuzzy rules; together with the state variables.

In this work, a tuning of the well known weighting parameters approach is proposed to optimize local and global approximation and modelling capability of the Takagi-Sugeno (T-S) fuzzy model to improve the choice of the performance index and minimize it. The main problem encountered is that the T-S identification method can not be applied when the membership functions are overlapped by pairs. This in turn restricts the application of the T-S method because this type of membership function has been widely used in control applications. The approach developed here can be considered as a generalized version of the T-S method. An inverted pendulum mounted on a cart is chosen to evaluate the robustness, effectiveness, accuracy and remarkable performance of the proposed estimation approach in comparison with the original T-S model. Simulation results indicate the potential, simplicity and generality of the estimation method and the robustness of the chattering reduction algorithm. In this paper, we prove that the proposed estimation algorithm converge the very fast, thereby making it very practical to use. The application of the proposed FLC-VSC shows that both alleviation of chattering and robust performance are achieved.
\end{abstract}

\section{INTRODUCTION}

Variable structure control (VSC) is naturally attractive to control engineers because its basic concepts are straight forward and have given satisfactory performance in many practical areas of industrial electronics. It has attracted interest recently because a fast calculation and switching action has been realized through the progress of micro and power electronics. Variable struchre control is characterized by its ability to deal with non-linearities, uncertainties of control systems, invariance to parameters, order reduction, fluctuations and disturbances, decoupling design procedure and its robustness.

The main VSC feature is to drive the state trajectory towards a sliding plan previously determined by computing the feedback control structure. It is desirable to reach simultaneously the sliding plane quickly, maintaining closeness to it, and to reduce the chattering between structures. However, reaching the sliding plane fast implies also a fast departure, unless a frequent switching between structures is allowed.

Motivated by the similarity between fuzzy logic controller (FLC) control rules and the VSC, the robustness of the FLC has been analyzed for nonlinear systems in this paper. As a result, the behavior of the fuzzy control system can be clarified and the relationship between the design parameters of the FLC and the tracking performances of the control system is addressed. The relationship is important since it gives guidance on the design parameters of the FLC to achieve the specified control performances [13-15, 38]

Sliding modes (SM) are used to determine best values for parameters in fuzzy control rules in which the robustness is inherent in the VSC with SM, thereby robustness in fuzzy control can be improved $[12,8,11,16,24,25,33]$.

In this paper, the design of a FLC-VSC is presented based on the Takagi-Sugeno (T-S). fuzzy model. We will show how the VSC-SM, the structure of which is based on mathematical analysis, can be made more appropriate for actual implementation by introduction of fuzzy rules.

It is well known that a robust a effective controller requires an accurate and efficient model. In [32], an interesting method is developed to identify nonlinear systems using input-output data. The identification process is divided in 
three steps; premise variables, MFs and consequent parameters. With respect to MFs, they a apply nonlinear programming technique using the complex method for the minimization of the performance index. The main problem encountered is that the T-S identification method can not be applied when the MFs are overlapped by pairs.

Nonlinear control systems based on the T-S model have attracted much attention during the last twenty years (e.g., see $[9,10]$. It provides a powerful solution for development of function approximation, systematic techniques to stability and design of fuzzy control systems in view of fruitful conventional control theory. They also allow relatively easy application of powerful learning techniques for their identification from data. This fuzzy method presents an alternative technique for representing complex nonlinear systems [43] and reduces the number of rules in modelling higher order nonlinear systems [9].

Takagi-Sugeno fuzzy models are proved to be universal function approximators as they are able to approximate any smooth nonlinear functions to any degree of accuracy in any convex compact region $[20,42,43]$. It was clearly shown that the number of fuzzy rules increases as the approximation error tends to zero [22], so it becomes difficult to make use of the universal approximation property of T-S fuzzy modelling for practical purposes. Moreover, if the number of rules is bounded, the resulting set of functions is nowhere dense in the space of approximated functions [35]. These conflicting objectives have motivated researchers to find a balance between the specified accuracy and the computational complexity of the resulting fuzzy model.

Recently, great interest has been shown to tensor product distributed compensation (TPDC) as an established controller design framework, that links TP model transformation and parallel distributed compensation (PDC) framework. The TP model transformation converts different models to a common representational form [4]. In [37] the approximation capabilities of TP model forms is analyzed, because the universal applicability of TPDC framework strongly relies on it. Consequently, in a class of control problems this property requires the usage of tradeoff techniques between the accuracy and the complexity of the TP form, which is easily feasible within the TPDC framework, unlike in analytic models.

In [31] the Higher Order Singular-ValueDecomposition (HOSVD) based canonical form of linear parameter varying models is presented and a model's most important invariant characteristics are developed. The numerical reconstructibility of the canonical form using a routinely executable tractable uniform method is analyzed. Convergency theorems for given numerical reconstruction constraint are presented.

In [2] a singular value-based method for reducing a given fuzzy rule set was discussed. The method conducts
SVD of the rule consequents and generates certain linear combinations of the original MFs to form new ones for the reduced set. The work characterizes MFs by the conditions of sum normalization ( $\mathrm{SN}$ ), nonnegativeness $(\mathrm{NN})$, and normality (NO). The proposed method is applicable regardless of the adopted inference paradigms. It discusses three applications of fuzzy reduction: fuzzy rule base with singleton support, fuzzy rule base with nonsingleton support, and the T-S model.

In [3] a transformation method is suggested to obtain a trade-off between the modelling complexity and accuracy of the T-S model. The suggested method is aimed at finding the minimal number of fuzzy rules for a given accuracy of a given T-S model. This trade-off is conceptually obtained by the discarding of those rules which have weak or no contribution to the output.

In [27], the authors proposed to obtain the best features of Mamdani and T-S models by using an affine global model with function approximation capabilities which maintains local interpretation. The suggested model is composed of variant coefficients which are independently governed by a zeroth order fuzzy inference system. This model may be interpreted as a generalization of the T-S model in which dynamics coefficients have been decoupled. They have shown that Mamdani and T-S models can be combined so that local and global interpretations are preserved.

Several results have been obtained regarding the identification of T-S models [6] and [20]. They are based upon two approaches, one is to linearize the original nonlinear system in various operating points when the model of the system is known, and the other is based on the input-output data collected from the original nonlinear system when its model is unknown. The authors in [6] use a fuzzy clustering method to identify T-S fuzzy models, including identification of the number of fuzzy rules and parameters of fuzzy MFs, and identification of parameters of local linear models by using a least squares method [30] to minimize the error between T-S models and the corresponding original nonlinear systems.

The authors in [20] suggest a method to improve the local and global approximation of the T-S model. However, this complicates the approximation to obtain both targets. It has been shown that constrained and regularized identification methods may improve interpretability of constituent local models as local linearizations, and locally weighted least squares method may explicitly address the trade-off between the local and global accuracy of T-S fuzzy models.

In [30] a new method of interval fuzzy model identification is developed. It combines a fuzzy identification methodology with some ideas from linear programming theory. The idea is extended to modelling the optimal lower and upper bound functions that define the band which contains all the measurement values. The method can be efficiently used in the case of the approximation of the nonlinear functions family. In [21], a fuzzy modelling method using the relevance 
vector learning mechanism (RVM) based on a kernel-based Bayesian estimation is introduced. The main aim is to find the best structure of the T-S fuzzy model for modelling nonlinear dynamic systems with measurement error. The number of rules and the parameter values of MFs can be found as optimizing the marginal likelihood of the RVM in the proposed fuzzy inference system.

In [22] the authors show that rule interpolation helps in reducing the identification complexity as it allows rule bases with gaps. It is suggested that only the minimal necessary number of rules remain which contain the essential information, and all other rules are replaced by the interpolation algorithm. This algorithm has drawbacks such as subnormal conclusion for certain configurations of the involved fuzzy sets and does not always lead to interpretable fuzzy MFs. In [36], a new technique for fuzzy rule interpolation was presented to combine the advantageous computational behavior of [22] and at the same time reduces the subnormality.

In $[1,18,19]$ new and efficient approaches are presented to improve the local and global estimation of T-S model. The aim is obtaining high function approximation accuracy and fast convergence. The main problem is that the T-S identification method can not be applied when the MFs are overlapped by pairs. The first approach uses the minimum norm method to search for an exact optimum solution at the expense of increasing complexity and computational cost. The second one is a simple and less computational method, based on weighting of parameters.

In spite of its advantageous properties in various approximation aspects and in complexity reduction, it was shown that $\alpha$-cut distance based fuzzy rule base interpolation has some essential deficiencies, for instance, it does not always result in immediately interpretable fuzzy MFs. In [5], an approach is presented to get rid of these disadvantages. It is based on the interpolation of relations instead of interpolating $\alpha$-cut distances, and which offers a way to derive a family of interpolation methods capable of eliminating some typical deficiencies of fuzzy rule interpolation techniques.

In [41] a generalized version of a previous Cartesian approach [40] for interpolating fuzzy rules of MFs with finite number of characteristic points is presented. Instead of representing MFs as points in Cartesian spaces, they now become elements in the space of a square, integrable function. Interpolation is thus made between the antecedent and consequent function spaces. This representation allows an extended class of MFs satisfying two monotonicity conditions to be accommodated in the interpolation process.

In [23], a study has outlined a new min-max approach to the fuzzy clustering, estimation, and identification with uncertain data. The proposed approach minimizes the worst-case effect of data uncertainties and modelling errors on estimation performance without making any statistical assumption and requiring a priori knowledge of uncertainties.
Several methods are used to deal with the problem of optimizing MFs, which are either derivative-based or derivative-free methods. The derivative-free approaches are desirable because they are more robust than derivative-based methods with respect to finding global minimum and with respect to a wide range of objective function and MFs types. The drawback is that they converge more slowly than derivative-based techniques [34]. On the other hand, derivative-based methods tend to converge to local minimums. In addition, they are limited to specific objective functions and types of inference and MFs. The most common approaches are: gradient descent [28], least squares [30], back propagation and Kalman filtering [29].

The rest of the paper is organized as follows. An itroduction to FLC-VSC is presented in Section II and a new method for chattering reduction is developed. In Section III, the design of a FLC-VSC controller is developed. Section IV deals with the estimation of the T-S fuzzy model. Sections $\mathrm{V}$ and VI entail an example of an inverted pendulum to demonstrate the potential, simplicity; and generality of the estimation method and the robustness of the chattering reduction algorithm. The conclusions of the effectiveness and validity of the proposed approach are explained in Section VII.

\section{VARIABLE STRUCTURE CONTROL}

The VSC is a combination of subsystems together with a suitable switching logic. In VSC, the design algorithm includes choosing the desired sliding functions which are formed by a choice of their parameters, as will be explained. Then a discontinuous control is found which assures the existence of the sliding modes at each point of the sliding plane $s(x)=0$. In the final stage, the control should drive the system states to the sliding plane. The robustness of VSC stems from the property that the behavior of the controlled system in the sliding mode only depends on the parameters of the sliding mode, not on the system parameters or any disturbances or fluctuations.

Let us design a VSC for $n^{\text {th }}$ order controllable system represented by:

$$
\dot{x}(t)=f(x(t), u(t))
$$

where,

$$
\begin{aligned}
& x=\left[\begin{array}{llll}
y(t) & \dot{y}(t) & \cdots & y^{(n-1)}(t)
\end{array}\right]^{T} \in \mathfrak{R}^{n} \\
& f: \mathfrak{R}^{n+1} \rightarrow \mathfrak{R}^{n}, \quad u \in R
\end{aligned}
$$

Supposing that the system can be modeled as follows:

$$
y^{(n)}(t)=f\left(y(t), \dot{y}(t), \cdots, y^{(n-1)}(t), u(t)\right)
$$


The structure of the VSC is determined by the sign of the vector valued function $s(x)$, which is defined to be the switching function which is assumed to be a linear one, i.e.,

$$
s(x)=C x, C \in \mathfrak{R}^{1 \times n}
$$

where

$$
C=\left[\begin{array}{llll}
c_{1} & c_{2} & \cdots & c_{n}
\end{array}\right], c_{n}=1
$$

where $C(x)$ is an arbitrary $(1 \times n)$ matrix chosen such that $s(x)=0$ defines a stable dynamic system of reduced order.

$$
s(x)=C x=c_{1} y+c_{2} \dot{y}+\cdots+c_{n-1} y^{(n-2)}+y^{(n-1)}=0
$$

Thus the characteristic polynomial:

$$
p_{s}(\lambda)=\lambda^{n-1}+c_{n-1} \lambda+\cdots+c_{2} \lambda+c_{1}
$$

will have all its roots in the negative left-side of the complex plane. The next step in the design of the VSC includes choosing the structure of the control to satisfy a reaching condition. There exist various structures of control algorithms which guarantee the existence of sliding modes. Sometimes, it is convenient to preassign the structure of the VSC and then determine the values of the controller gain. The design of VSC can proceed with the structure of the control $u(x)$ as free or preassigned. In the free structure approach, the control structure can be solved by constraining the switching function to any one of various reaching conditions mentioned in [39]:

$$
\begin{aligned}
& \text { If } s(x)<0 \text { then } \dot{s}(x)>0 \\
& \text { If } s(x)>0 \text { then } \dot{s}(x)<0
\end{aligned}
$$

In this article, we have proposed the following switching condition:

$$
\begin{aligned}
& \text { If } s(x)>0 \text { then } \dot{s}(x)=K \\
& \text { If } s(x)<0 \text { then } \dot{s}(x)=-K
\end{aligned}
$$

If we suppose that the system can be modeled as follows:

$$
\begin{aligned}
& {\left[\begin{array}{c}
\dot{x}_{1} \\
\dot{x}_{2} \\
\vdots \\
\dot{x}_{n-1} \\
\dot{x}_{n}
\end{array}\right]=\left[\begin{array}{c}
0 \\
0 \\
\vdots \\
0 \\
a_{0}
\end{array}\right]+\left[\begin{array}{ccccc}
0 & 1 & 0 & \cdots & 0 \\
0 & 0 & 1 & \cdots & \vdots \\
\vdots & \vdots & \ddots & \ddots & 0 \\
0 & 0 & 0 & \cdots & 1 \\
a_{1} & a_{2} & a_{3} & \cdots & a_{n}
\end{array}\right]} \\
& {\left[\begin{array}{c}
x_{1} \\
x_{2} \\
\vdots \\
x_{n-1} \\
x_{n}
\end{array}\right]+\left[\begin{array}{c}
0 \\
0 \\
\vdots \\
0 \\
s(x)=C x(t)=C\left(A_{0}+A x(t)+B u(t)\right) \\
=b_{n}
\end{array}\right] u=A_{0}+A x(t)+B u(t)}
\end{aligned}
$$

$$
C B u(t)=\alpha-a_{0}-C A x(t)
$$

it can be observed that:

$$
C B=\left[\begin{array}{lllll}
c_{1} & c_{2} & \ldots & c_{n-1} & 1
\end{array}\right]\left[\begin{array}{c}
0 \\
0 \\
\vdots \\
0 \\
b_{n}
\end{array}\right]=b_{n}
$$

CB will be always invertibe if $b_{n} \neq 0$ which is a necessary condition for the system to be controllable.

$$
u(t)=[C B]^{-1}\left(\alpha-a_{0}-C A x(t)\right)=\frac{1}{b_{n}}\left(\alpha-a_{0}-C A x(t)\right)
$$

and the VSC will be:

$$
\begin{aligned}
& \text { If } s(x)<0 \text { then } u(t)=\frac{1}{b_{n}}\left(K-a_{0}-C A x(t)\right) \\
& \text { If } s(x)>0 \text { then } u(t)=\frac{1}{b_{n}}\left(-K-a_{0}-C A x(t)\right)
\end{aligned}
$$

Example. Let us analyze the following unstable linear system:

$$
\begin{aligned}
& Y(s)=\frac{1}{s^{2}-2 s+5} U(s) \\
& \dot{x}(t)=\left[\begin{array}{cc}
0 & 1 \\
-5 & 2
\end{array}\right] x(t)+\left[\begin{array}{l}
0 \\
1
\end{array}\right] u(t) \\
& s(x)=\left[\begin{array}{ll}
1 & 1
\end{array}\right] x(t)
\end{aligned}
$$

$$
p_{s}(\lambda)=\lambda+1
$$$$
K=10
$$

The control action will be:

$$
\begin{aligned}
u & \left.\left.=-\left[\begin{array}{ll}
1 & 1
\end{array}\right]\left[\begin{array}{l}
0 \\
1
\end{array}\right]\right]^{-1}\left(\begin{array}{ll}
1 & 1
\end{array}\right]\left[\begin{array}{cc}
0 & 1 \\
-5 & 2
\end{array}\right] x(t)-\alpha\right) \\
& =-[-53] x(t)+\alpha=5 x_{1}(t)-3 x_{2}(t)+\alpha
\end{aligned}
$$

Thus the control action will be,

$$
\begin{aligned}
& \text { If } s(x)<0 \text { then } u(t)=5 x_{1}(t)-3 x_{2}(t)+10 \\
& \text { If } s(x)>0 \text { then } u(t)=5 x_{1}(t)-3 x_{2}(t)-10
\end{aligned}
$$




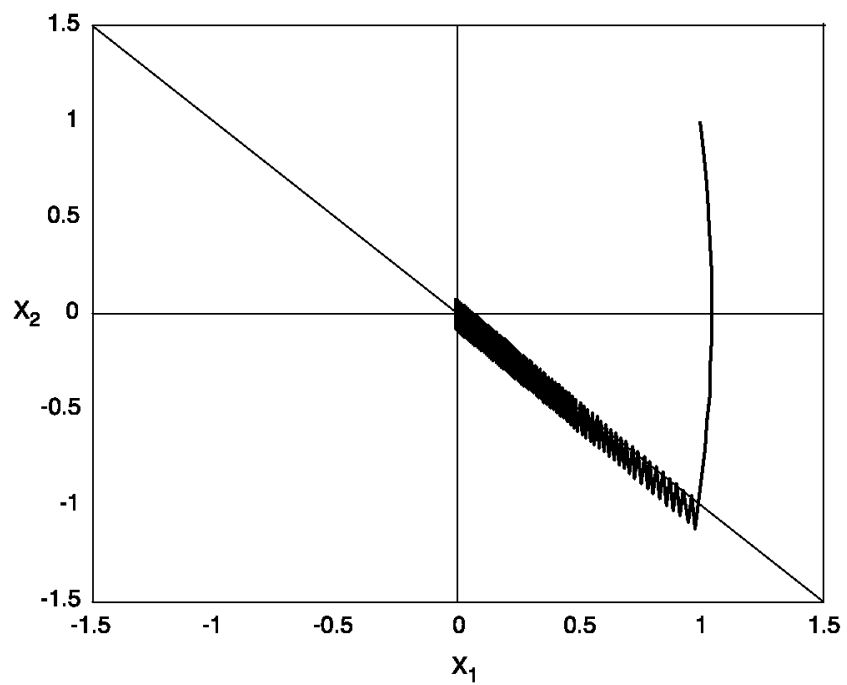

Fig. 1. Chattering effect.

Fig. 1 shows the chattering effect assuming that the simulation is carried out with a sampling time of 0.01 seconds.

\subsection{Chattering reduction}

As mentioned before, the main drawback of sliding mode control is the chattering problem resulting from the switching from one value to another. In this paper, we present the idea of the equivalent control which yields the ideal sliding mode, defined as the one found by recognizing that $s(x)=0$ is a necessary and sufficient condition for the state trajectory to stay on the switching surface $s(x)=0$.

In order to overcome the chattering problem, a boundary layer is introduced around the switching surface.The resultant controller is a combination of the discontinuous one used to create the sliding mode outside the layer and applying the equivalent control method inside this layer. Therefore, it is suggested in this work to switch from the sliding model control explained previovsly to the equivalent control according to the position of the state trajectory. The necessary and sufficient condition $s(x)=0$ required for the equivalent control to exist can be modelled as follows:

$$
\text { If } s(x)=0 \text { then } s(x)=0
$$

Thus, the VSC will be:

$$
\begin{aligned}
& \text { If } s(x)<0 \text { then } \dot{s}(x)>0 \\
& \text { If } s(x)=0 \text { then } \dot{s}(x)=0 \\
& \text { If } s(x)>0 \text { then } \dot{s}(x)<0
\end{aligned}
$$

The problem is that the second condition will almost never be fulfilled. The solution proposed here for this problem is

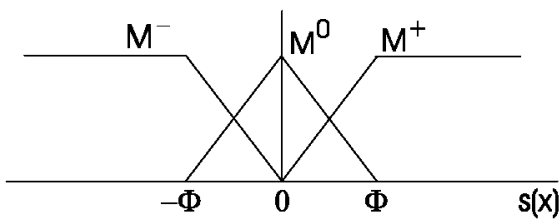

Fig. 2. Fuzzy sets of the switching surface.

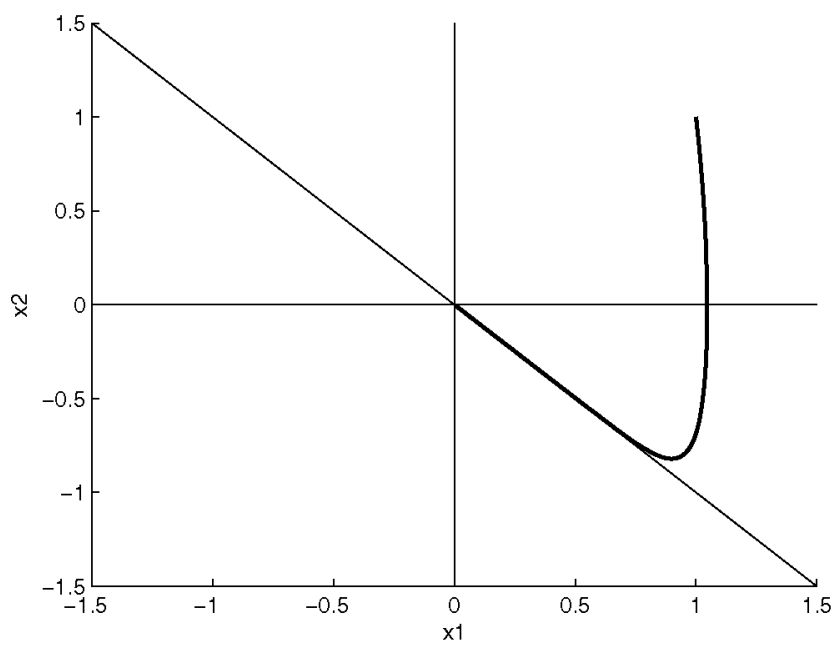

Fig. 3. Chattering elimination applying the proposed fuzzy algorithm.

converting the VSC in fuzzy control by defining three fuzzy sets for $\mathrm{s}(\mathrm{x})$ as shown in Fig. 2. The resultant fuzzy VSC is:

$$
\begin{aligned}
& \text { If } s(x) \text { is } M^{-} \text {then } \dot{s}(x)>0 \\
& \text { If } s(x) \text { is } M^{0} \text { then } \dot{s}(x)=0 \\
& \text { If } s(x) \text { is } M^{+} \text {then } \dot{s}(x)<0
\end{aligned}
$$

If the system is an affine one then the control action will be:

$$
\begin{aligned}
& \text { If } s(x) \text { is } M^{-} \text {then } u(t)=\frac{1}{b_{n}}\left(K-a_{0}-C A x(t)\right) \\
& \text { If } s(x) \text { is } M^{0} \text { then } u(t)=\frac{1}{b_{n}}\left(-a_{0}-C A x(t)\right) \\
& \text { If } s(x) \text { is } M^{+} \text {then } u(t)=\frac{1}{b_{n}}\left(-K-a_{0}-C A x(t)\right)
\end{aligned}
$$

Now, the proposed fuzzy control is applied for the same system in the previous example with the same sampling time and the fuzzy sets shown in Fig. 2 with $\Phi=1$. The effect of the chattering can be clearly seen (see Fig. 1). The mean square distance of the system state trajectory to the switching surface for the final five seconds is 0.0089 , while the same distance is noticeably reduced by applying the proposed anti chattering fuzzy controller to $9.3769 \mathrm{e}-012$ (see Fig. 3 which obviously shows the effectiveness of suggested controller. In this paper, fuzzy inference is applied to switch from the 
discontinuous controller to the equivalent one by regarding the distance from the switching hyperplane as a variable of the premise of the control laws.

\section{PROPOSED CONTROLLER (FLC-VSC)}

There exist various techniques to design the FLC-VSC. In most of the works carried out in this field [17], [26], the fuzzy system is described as follows:

$$
\dot{x}=A(x) x+B(x) u
$$

This means that the non linear fuzzy system is linearized with respect to the origin in each IF-THEN rule, which means that the consequent part of each rule is a linear function with zero independent term. This will in turn reduce the accuracy of approximating non linear systems. Moreover, in linear control theory, the affine term does not affect the dynamics of the system, rather the input to it. In the case of fuzzy control, the blending of the affine term of each rule will no longer be a constant but a function of the variables of the system and thus affects the dynamics of the resultant system. In this study, a design of FLC-VSC is presented based on the T-S fuzzy model [32], taking into account the effect of the affine term in both the fuzzy system and controller.

\subsection{Fuzzy logic controller based variable structure controller (FLC-VSC)}

Let us consider the problem of designing a fuzzy controller based on variable structure with VSC theory. It should be mentioned that the resultant feedback controlled system is an approximation of the nonlinear original system.

Let the $\left(i_{1} \ldots i_{n}\right)^{\text {th }}$ rule of the T-S model be represented as:

$$
\begin{aligned}
& S^{\left(i_{1} \ldots i_{n}\right)} \text { : If } x_{1} \text { is } M_{1}^{i_{1}} \text { and } x_{2} M_{2}^{i_{2}} \text { and } \ldots \\
& \text { and } x_{n} \text { is } M_{n}^{i_{n}} \text { then } \dot{x}=a_{0}^{\left(i_{1} \ldots i_{n}\right)}+A^{\left(i_{1} \ldots i_{n}\right)} x+B^{\left(i_{1} \ldots i_{n}\right)} u
\end{aligned}
$$

where $M_{1}^{i_{1}}\left(i_{1}=1,2, \ldots, r_{1}\right)$ are fuzzy sets for $X_{1}, M_{2}^{i_{2}}\left(i_{2}=1\right.$, $\left.2, \ldots, \mathbf{r}_{2}\right)$ are fuzzy sets for $X_{2}, M_{n}^{i_{n}}\left(i_{n}=1,2, \ldots, r_{n}\right)$ are fuzzy sets for $X_{n}$. Therefore the complete fuzzy system has $r_{1} \times r_{2} \times \ldots r_{n}$ rules. The MFs of the fuzzy system are overlapped by pairs.

The state vector is:

$$
x^{T}=\left[\begin{array}{llll}
x_{1} & x_{2} & \ldots & x_{n}
\end{array}\right]
$$

of $\mathrm{n}$ dimension, and $\mathbf{u}$ is a scalar input. The vectors and matrices in these rules are described in a canonical controllable form:

$$
\begin{aligned}
& a_{0}^{\left(i_{1} \ldots i_{n}\right)}=\left[\begin{array}{c}
0 \\
\vdots \\
0 \\
a_{0}^{\left(i_{1} \ldots i_{n}\right)}
\end{array}\right] \\
& A^{\left(i_{1} \ldots i_{n}\right)}=\left[\begin{array}{cccc}
0 & 0 & \cdots & 0 \\
0 & 1 & \ddots & \vdots \\
\vdots & \ddots & \ddots & 0 \\
0 & \ldots & 0 & 1 \\
a_{1}^{\left(i_{1} \ldots i_{n}\right)} & \cdots & a_{n-1}^{\left(i_{1} \ldots i_{n}\right)} & a_{n}^{\left(i_{1} \ldots i_{n}\right)}
\end{array}\right] \\
& B^{\left(i_{1} \ldots i_{n}\right)}=\left[\begin{array}{c}
0 \\
\vdots \\
0 \\
b_{n}^{\left(i_{1} \ldots i_{n}\right)}
\end{array}\right]
\end{aligned}
$$

The same MFs for the fuzzy system are used for the FLC-VSC controller. The fuzzy controller action for each systems rule can be written as follows:

$$
\begin{aligned}
& R^{\left(i_{1} \ldots i_{n},-\right)}: \text { If } x_{1} \text { is } M_{1}^{i_{1}} \text { and } x_{2} \text { is } M_{2}^{i_{2}} \text { and } \ldots \\
& \text { and } x_{n} \text { is } M_{n}^{i_{n}} \text { and } s(x) \text { is } M^{-} \text {then } \\
& u(t)=\frac{1}{b_{n}^{\left(i_{1} \ldots i_{n}\right)}}\left(K-a_{0}^{\left(i_{1} \ldots i_{n}\right)}-C A^{\left(i_{1} \ldots i_{n}\right)} x(t)\right) \\
& R^{\left(i_{1} \ldots i_{n}, 0\right)}: \text { If } x_{1} \text { is } M_{1}^{i_{1}} \text { and } x_{2} \text { is } M_{2}^{i_{2}} \text { and } \ldots \\
& \text { and } x_{n} \text { is } M_{n}^{i_{n}} \text { and } s(x) \text { is } M^{0} \text { then } \\
& u(t)=\frac{1}{b_{n}^{\left(i_{1} \ldots i_{n}\right)}}\left(-a_{0}^{\left(i_{1} \ldots i_{n}\right)}-C A^{\left(i_{1} \ldots i_{n}\right)} x(t)\right) \\
& R^{\left(i_{1} \ldots i_{n},+\right)}: \text { If } x_{1} \text { is } M_{1}^{i_{1}} \text { and } x_{2} \text { is } M_{2}^{i_{2}} \text { and } \ldots \\
& \text { and } x_{n} \text { is } M_{n}^{i_{n}} \text { and } s(x) \text { is } M^{+} \text {then } \\
& u(t)=\frac{1}{b_{n}^{\left(i_{1} \ldots i_{n}\right)}}\left(-K-a_{0}^{\left(i_{1} \ldots i_{n}\right)}-C A^{\left(i_{1} \ldots i_{n}\right)} x(t)\right)
\end{aligned}
$$

\section{ESTIMATION OF FUZZY T-S MODEL'S PARAMETERS}

The T-S model [32] is based on estimating the nonlinear system parameters minimizing a quadratic performance index. The method is based on the identification of functions of the following form: 


$$
\begin{aligned}
& f: \Re^{n} \rightarrow \Re \\
& y=f\left(v_{1}, v_{2}, \ldots, v_{n}\right)
\end{aligned}
$$

Each IF-THEN rule for an $n^{\text {th }}$ order system can be written as follows:

$$
\begin{aligned}
& S^{\left(i_{1} \ldots i_{n}\right)}: \text { If } v_{1} \text { is } M_{1}^{i_{1}} \text { and } \ldots v_{n} \text { is } M_{n}^{i_{n}} \text { then } \\
& y=p_{0}^{\left(i_{1} \ldots i_{n}\right)}+p_{1}^{\left(i_{1} \ldots i_{n}\right)} v_{1}+\ldots+p_{n}^{\left(i_{1} \ldots i_{n}\right)} v_{n}
\end{aligned}
$$

where the fuzzy estimation of the output is:

$$
\begin{aligned}
\hat{y}= & \sum_{i_{1}=1}^{r_{1}} \ldots \sum_{i_{n}=1}^{r_{n}} \beta^{\left(i_{1} \ldots i_{n}\right)}(v)\left[p_{0}^{\left(i_{1} \ldots i_{n}\right)}\right. \\
& \left.+p_{1}^{\left(i_{1} \ldots i_{n}\right)} v_{1}+\ldots+p_{n}^{\left(i_{1} \ldots i_{n}\right)} v_{n}\right]
\end{aligned}
$$

where,

$$
\beta_{k}^{\left(i_{1} \ldots i_{n}\right)}=\frac{w^{\left(i_{1} \ldots i_{n}\right)}\left(v_{k}\right)}{\sum_{i_{1}=1}^{n_{1}} \ldots \sum_{i_{n}=1}^{n_{n}} w^{\left(i_{1} \ldots i_{n}\right)}\left(v_{k}\right)}
$$

and

$$
w^{\left(i_{1} \ldots i_{n}\right)}(v)=\prod_{l=1}^{n} \mu_{i i_{l}}\left(v_{l}\right)
$$

being $\mu_{j i_{j}}\left(v_{j}\right)$ the membership function that corresponds to the fuzzy set $M_{j}^{i_{j}}$.

Let $\left\{v_{l k}, v_{2 k}, \ldots, v_{n k}, y_{k}\right\}$ be a set of input/output system samples. The parameters of the fuzzy system can be calculated as a result of minimizing a quadratic performance index:

$$
J=\sum_{k=1}^{m}\left(y_{k}-\hat{y}_{k}\right)^{2}=\|Y-X P\|^{2}
$$

where

$$
\begin{aligned}
& Y=\left[\begin{array}{llll}
y_{1} & y_{2} & \ldots & y_{m}
\end{array}\right]^{T} \\
& P=\left[\begin{array}{lll}
p_{0}^{(1 \ldots 1)} p_{1}^{(1 \ldots 1)} p_{2}^{(1 \ldots 1)} & \ldots & p_{n}^{(1 \ldots 1)} \ldots \\
p_{0}^{\left(r_{1} \ldots n_{n}\right)} \ldots p_{n}^{\left(r_{1} \ldots r_{n}\right)} & &
\end{array}\right]^{T}
\end{aligned}
$$

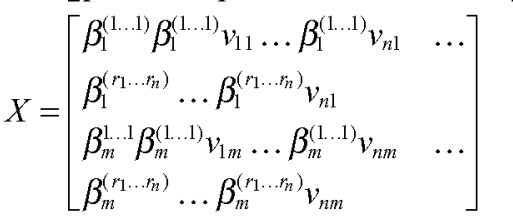

If $\mathrm{X}$ is a matrix of full rank, the solution is obtained as follows:

$$
\begin{aligned}
J & =\|Y-X P\|^{2}=(Y-X P)^{T}(Y-X P) \\
\nabla J & =X^{T}(Y-X P)=X^{T} Y-X^{T} X P=0 \\
P & =\left(X^{T} X\right)^{-1} X^{T} Y
\end{aligned}
$$

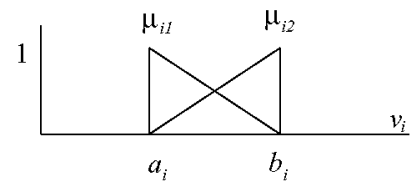

Fig. 4. Membership functions of the fuzzy system.

The method proposed in [32] poses serious problems as it can not be applied in the most common case where the MFs are those shown in Fig. 4. The MFs $\mu_{i 1}\left(v_{i}\right)=\frac{b_{i}-v_{i}}{b_{i}-a_{i}}$ and $\mu_{i 2}\left(v_{i}\right)=\frac{v_{i}-a_{i}}{b_{i}-a_{i}}$ are defined in an interval $\left[a_{i}, b_{i}\right]$ which should verify:

$$
\begin{array}{ll}
\mu_{i 1}\left(a_{i}\right)=1 & \mu_{i 1}\left(b_{i}\right)=0 \\
\mu_{i 2}\left(a_{i}\right)=0 & \mu_{i 2}\left(b_{i}\right)=1 \\
\mu_{i 1}\left(v_{i}\right)+\mu_{i 2}\left(v_{i}\right)=1 &
\end{array}
$$

For this case, which is widely used, it can be easily demonstrated that the matrix $X$ is not of full rank and therefore $X^{T} X$ is not invertible, which makes the mentioned method of T-S invalid. A detailed proof can be found in $[1,18,19]$.

In the case when the matrix $X$ is not of full rank, an effective approach with few computational effort, based on the well-known parameters' weighting method, is presented in $[1,19]$. Several nonlinear examples are included to show the validity and accuracy of the proposed method. The main target is to improve the choice of the performance index and minimize it. It is characterized by extending the objective function by including a weighting $\gamma$ of the norm of $P$ vector.

$$
J=\sum_{k=1}^{m}\left(y_{k}-\hat{y}_{k}\right)^{2}+\gamma^{2} \sum_{j} p_{j}^{2}=\|Y-X P\|^{2}+\gamma^{2}\|P\|^{2}
$$

This can be rewritten as follows:

$$
\begin{aligned}
J & =\|Y-X P\|^{2}+\gamma^{2}\|P\|^{2} \\
& =\left\|\left[\begin{array}{l}
Y \\
0
\end{array}\right]-\left[\begin{array}{c}
X \\
\gamma I
\end{array}\right] P\right\|^{2} \\
& =\left\|Y_{a}-X_{a} P\right\|^{2}
\end{aligned}
$$

Now the extended matrix $X_{a}$ is of full rank, and the vector $P$ can be computed as:

$$
P=\left(X_{a}^{T} X_{a}\right)^{-1} X_{a}^{T} Y_{a}
$$

\subsection{Parameter tuning using the parameters weighting method}

The parameter weighting method proposed in [1] and [19] can also be used for parameters tuning of the T-S 
model from local parameters obtained through the identification of a system in an operating region or from any physical input/output data. The tuning is required for control objectives. What is firstly required is to obtain rules of physical significance. Secondly, the overlapping rules should be minimized. We suppose that in this case we have a first estimation

$$
P_{0}=\left[p_{0}^{0} p_{1}^{0} p_{2}^{0} \ldots p_{n}^{0}\right]^{T}
$$

of the T-S model parameters. In order to obtain such an estimation, the classical least square method can be used around the equilibrium point. The objective is to obtain a global approximation of the system:

$$
\hat{y}=p_{0}^{0}+p_{1}^{0} v_{1}+p_{2}^{0} v_{2}+\ldots+p_{n}^{0} v_{n}
$$

Let us analyze a set of input/output system samples $\left\{v_{1 k}, v_{2 k}\right.$, $\left.\ldots, v_{n k}, y_{k}\right\}$. The parameters of the global approximation can be calculated by minimizing the following quadratic performance index:

$$
J=\sum_{k=1}^{m}\left(y_{k}-\hat{y}_{k}\right)^{2}=\left\|Y-X_{g} P_{0}\right\|^{2}
$$

where

$$
\begin{aligned}
Y & =\left[\begin{array}{lllll}
y_{1} & y_{2} & y_{3} & \ldots & y_{m}
\end{array}\right]^{T} \\
P_{0} & =\left[\begin{array}{lllll}
p_{0}^{0} & p_{1}^{0} & p_{2}^{0} & \ldots & p_{n}^{0}
\end{array}\right]^{T} \\
X_{g} & =\left[\begin{array}{lllll}
1 & v_{11} & v_{21} & \ldots & v_{n 1} \\
1 & v_{12} & v_{22} & \ldots & v_{n 2} \\
\vdots & \vdots & \vdots & \ddots & \vdots \\
1 & v_{1 m} & v_{2 m} & \ldots & v_{n m}
\end{array}\right]
\end{aligned}
$$

In this case, if we select a sufficient number of points distributed in the region where it is required to obtain the approximation, then the matrix $X_{g}$ will be of a full rank and therefore, the solution becomes unique, and can be calculated as follows:

$$
P_{0}=\left(X_{g}^{T} X_{g}\right)^{-1} X_{g}^{T} Y
$$

This first approximation can be utilized as reference parameters for all the subsystems. Then, the parameters' vector of the fuzzy model can be obtained minimizing:

$$
\begin{aligned}
J & =\sum_{k=1}^{m}\left(y_{k}-\hat{y}_{k}\right)^{2}+\gamma^{2} \sum_{i_{1}=1}^{r_{1}} \ldots \sum_{i_{n}=1}^{r_{n}} \sum_{j=0}^{n}\left(p_{j}^{0}-p_{j}^{\left(i_{1} \ldots i_{n}\right)}\right)^{2} \\
& =\|Y-X p\|^{2}+\gamma^{2}\left\|p_{0}-p\right\|^{2} \\
& =\left\|\left[\begin{array}{l}
Y \\
\gamma p_{0}
\end{array}\right]-\left[\begin{array}{l}
X \\
\gamma I
\end{array}\right] p\right\|^{2}=\left\|Y_{a}-X_{a} p\right\|^{2}
\end{aligned}
$$

where

$$
p_{0}=\underbrace{\left[P_{0} P_{0} \ldots P_{0}\right]^{T}}_{r_{1} \cdot r_{2} \ldots p_{n}}
$$

In this case, the factor $\gamma$ represents the degree of confidence of the parameters initially estimated. In a similar way to the previous case, different weight factors of $\gamma_{j}^{\left(i_{1} \ldots i_{n}\right)}$ can be used to each one of the parameters $p_{j}^{\left(i_{1} \ldots i_{n}\right)}$ depending on the reliability of the initial parameter $p_{j}^{0}$ in the specific rule.

$$
\begin{aligned}
J & =\sum_{k=1}^{m}\left(y_{k}-\hat{y}_{k}\right)^{2}+\sum_{i_{1}=1}^{r_{1}} \ldots \sum_{i_{n}=1}^{r_{n}} \sum_{j=0}^{n} \gamma_{j}^{\left(i_{1} \ldots i_{n}\right) 2}\left(p_{j}^{0}-p_{j}^{\left(i_{1} \ldots i_{n}\right)}\right)^{2} \\
& =\|Y-X p\|^{2}+\left\|\Gamma\left(p_{0}-p\right)\right\|^{2} \\
& =\left\|\left[\begin{array}{l}
Y \\
\Gamma p_{0}
\end{array}\right]-\left[\begin{array}{l}
X \\
\Gamma
\end{array}\right] p\right\|^{2}=\left\|Y_{a}-X_{a} p\right\|^{2}
\end{aligned}
$$

where $\Gamma$ is a diagonal matrix with the weight factor $\gamma_{j}^{\left(i_{1} \ldots i_{n}\right)}$. It is not necessary to apply this process for all the parameters. If the values of some of them are known, they can be fixed beforehand, or we can assign them a weighting factor $\gamma_{j}^{\left(i_{1} \ldots i_{n}\right)}$ comparatively high.

By applying the proposed weighting of parameters approach for various values of $\gamma$, it is aimed to examine the trade-off between the accuracy of the identification of the model and the condition number.

\subsection{Application of the proposed estimation method for T-S dynamic systems}

The main aim is applying the parameters' weighting method mentioned above for the identification of system parameters given by (15) and (16). This is equivalent to identifying a system described by (17) which is transformed to the following equation:

$$
\dot{x}_{n}=f\left(x_{1}, x_{2}, \ldots, x_{n}, u\right)
$$

The objective is to find an appropriate T-S model which allows us to obtain the control action $u$ as a function of the state variable $x$. Therefore, we aim at getting the best T-S model with the premise partly depending on state variable $x$ and the consequent part that depends on both state variable $x$ and control action $u$. Thus, as can be seen from (17) that $y$ is substituted in (34) by $\dot{x}_{n}$ and the $v$ vector is substituted by the state vector $x$ and the input $u$. This adaptation between (17) and (34) comes from the fact that the system variables do not depend on $u$. So, the most suitable form to obtain $u$ is the one given in (35) where it can be observed that the control action appears only in the consequent part and not in premise part as desired. $a_{0}^{\left(i_{1} \ldots i_{n}\right)}, A^{\left(i_{1} \ldots i_{n}\right)}$ and $B^{\left(i_{1} \ldots i_{n}\right)}$ are determined by T-S model given by IF-THEN rules as follows: 


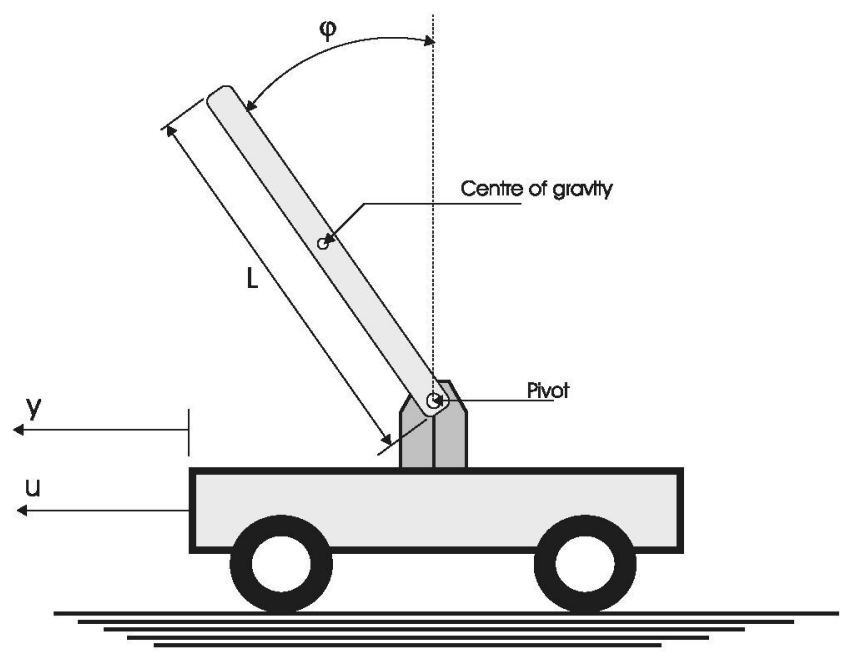

Fig. 5. Inverted pendulum system.

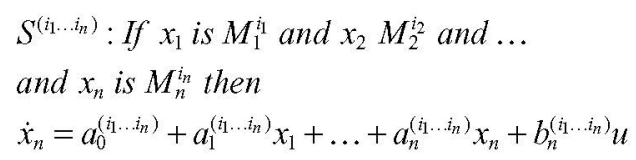

and the new vector of parameters to be determined becomes:

$$
p=\left[\begin{array}{lllll}
a_{0}^{(1 \ldots 1)} & a_{1}^{(1 \ldots 1)} & \ldots & a_{n}^{(1 \ldots 1)} & b_{n}^{(1 \ldots 1)} \ldots \\
a_{0}^{\left(r_{1} \ldots r_{n}\right)} & a_{1}^{\left(r_{1} \ldots r_{n}\right)} & \ldots & a_{n}^{\left(r_{1} \ldots r_{n}\right)} & b_{n}^{\left(r_{1} \ldots r_{n}\right)}
\end{array}\right]^{T}
$$

\section{ILLUSTRATIVE EXAMPLE}

In this section, the proposed parameters' weighting method mentioned above is used for the estimation of an inverted pendulum. Then, the suggested FLC-VSC is applied to control it.

\subsection{Estimation of an inverted pendulum using the proposed parameter weighting method}

Consider the problem of estimating an inverted pendulum (see Fig. 5) using the above mentioned estimation methods. The inverted pendulum can be represented as follows:

$$
\ddot{\theta}=\frac{g \sin \theta-\cos \theta\left(\frac{u+m l \dot{\theta}^{2} \sin \theta}{M+m}\right)}{l\left(\frac{4}{3}-\frac{m \cos ^{2} \theta}{M+m}\right)}
$$

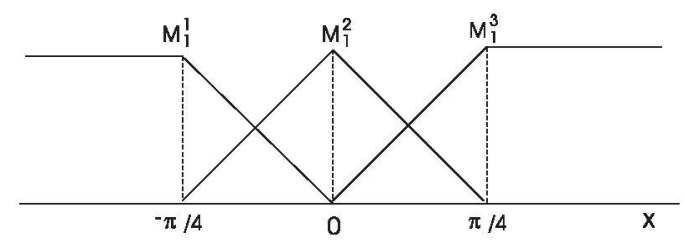

Fig. 6. Membership functions for the angle $x$ of the inverted pendulum.

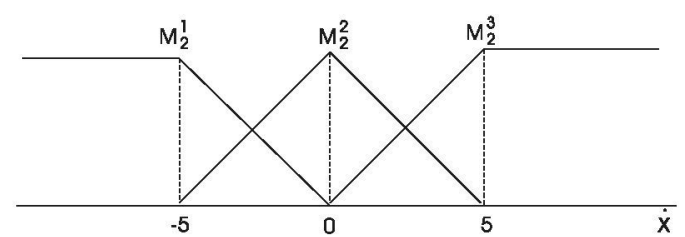

Fig. 7. Membership functions for the angular velocity of the inverted pendulum.

Where $\theta$ denotes the angular position (in radians) deviated from the equilibrium position (vertical axis) of the pendulum and $\dot{\theta}$ is the angular velocity, $\mathrm{g}$ (gravity acceleration) $=9.8 \frac{\mathrm{m}}{\mathrm{sec}^{2}}, \quad M$ (mass) of the cart $=1 \mathrm{~kg}, m$ (mass) of the pole $=0.1 \mathrm{~kg}, 1$ is the distance from the center of the mass $(\mathrm{m})$ of the pole to the cart $=0.5 \mathrm{~m}$. Assuming that $\mathrm{X}_{1}=\theta, \mathrm{x}_{2}=\dot{\theta}$ and $u$ is an external force applied to the cart in the horizontal direction, then (36) can be rewritten in state space form as follows:

$$
\begin{aligned}
& x_{1}=\theta \\
& \dot{x}_{1}=x_{2} \\
& x_{2}=\dot{\theta} \\
& \dot{x}_{2}=\frac{g \sin \left(x_{1}\right)-\cos \left(x_{1}\right)\left(\frac{u+m l x_{2}^{2} \sin \left(x_{1}\right)}{M+m}\right)}{l\left(\frac{4}{3}-\frac{m \cos ^{2}\left(x_{1}\right)}{M+m}\right)}
\end{aligned}
$$

Firstly, the model of the inverted pendulum is estimated in three operation points for both the angle and its derivative. The universe of discourse of the angle and the angular velocity $\left[\frac{-\pi}{4}, \frac{\pi}{4}\right]$ rad. and $[-5,5] \frac{\mathrm{rad}}{\mathrm{sec}}$, respectively. Both MFs for the angle $\mathrm{x}$ and its derivative $\dot{x}$ are shown in Figs 6 and 7 respectively. If we apply the T-S method directly to this example, then the condition number of the matrix $\mathrm{X}$ is $3.4148 \mathrm{e}+015$, which shows clearly a non reliable result. Using the parameter weighting method with weighting factor $\gamma=0.01$, the the inverted pendulum fuzzy model can be represented as follows: 


$$
\begin{aligned}
& S^{11}: \text { If }\left(x_{1} \text { is } M_{1}^{1}\right) \text { and }\left(x_{2} \text { is } M_{2}^{1}\right) \text { then } \\
& \dot{x}_{2}=-8.1994+3.4151 x_{1}-0.2006 x_{2}-1.0443 u \\
& S^{12}: \text { If }\left(x_{1} \text { is } M_{1}^{1}\right) \text { and }\left(x_{2} \text { is } M_{2}^{2}\right) \text { then } \\
& \dot{x}_{2}=-8.3766+3.0426 x_{1}+0.0000 x_{2}-1.0443 u \\
& S^{13}: \text { If }\left(x_{1} \text { is } M_{1}^{1}\right) \text { and }\left(x_{2} \text { is } M_{2}^{3}\right) \text { then } \\
& \dot{x}_{2}=-8.1994+3.4151 x_{1}+0.2006 x_{2}-1.0443 u \\
& S^{21}: \text { If }\left(x_{1} \text { is } M_{1}^{2}\right) \text { and }\left(x_{2} \text { is } M_{2}^{1}\right) \text { then } \\
& \dot{x}_{2}=-0.0251+5.5416 x_{1}-0.0085 x_{2}-1.5332 u \\
& S^{22}: \text { If }\left(x_{1} \text { is } M_{1}^{2}\right) \text { and }\left(x_{2} \text { is } M_{2}^{2}\right) \text { then } \\
& \dot{x}_{2}=0.0225+6.1796 x_{1}-0.0000 x_{2}-1.5332 u \\
& S^{23}: \text { If }\left(x_{1} \text { is } M_{1}^{2}\right) \text { and }\left(x_{2} \text { is } M_{2}^{3}\right) \text { then } \\
& \dot{x}_{2}=-0.0251+5.5416 x_{1}+0.0085 x_{2}-1.5332 u \\
& S^{31}: \text { If }\left(x_{1} \text { is } M_{1}^{3}\right) \text { and }\left(x_{2} \text { is } M_{2}^{1}\right) \text { then } \\
& \dot{x}_{2}=8.1236+3.8634 x_{1}+0.2363 x_{2}-1.0903 u \\
& S^{32}: \text { If }\left(x_{1} \text { is } M_{1}^{3}\right) \text { and }\left(x_{2} \text { is } M_{2}^{2}\right) \text { then } \\
& \dot{x}_{2}=8.0313+3.6645 x_{1}-0.0000 x_{2}-1.0903 u \\
& S^{33}: \text { If }\left(x_{1} \text { is } M_{1}^{3}\right) \text { and }\left(x_{2} \text { is } M_{2}^{3}\right) \text { then } \\
& \dot{x}_{2}=8.1236+3.8634 x_{1}-0.2363 x_{2}-1.0903
\end{aligned}
$$

The resultant mean square error from this approximation is 0.0013 . In this case, the condition number of the extended matrix $X_{a}$ becomes $1.4569 \mathrm{e}+004$, thus improving the reliability of results. By using the identification with the classical minimum square method in an interval close to the equlibrium point

$$
x_{1} \in\left[\frac{-\pi}{4}, \frac{\pi}{4}\right] \quad \mathrm{x}_{2} \in[-2.5,2.5]
$$

The linear model of the system in this interval is:

$$
\dot{x}_{2}=0.0092+15.2665 x_{1}-0.0000 x_{2}-1.4187 u
$$

This approxiamtion gives us an idea of the quantative relation among various variables. Comparing these parameters with those obtanied in the previous fuzzy model, it is evident that its values are, in general, far enough from its physical ones. Moreover, if we compare the parameters of various adjacent subsystems, it can be clearlied verify that they are quite different. The tuning of parameters method is applied; taking as a reference the parameters obtained through minimum square method with a weighting factor $\gamma=0.01^{*} n p$, where np is the number of samples used in the identification. As it can be seen when $a_{0}^{22} \neq 0$ it does not fulfil an important characteristic of the existence of an instable equilibrium point, which is also the objective of control $x_{1}=x_{2}=u=0$. In order that the point $x_{1}=x_{2}=u=0$ becomes an equlibrium one in the fuzzy model, $a_{0}^{22}$ should be zero. To achieve this target, we can either fix the value of this parameter or assign it a very large weighting factor as explained in Section 4.1. In this case, a weighting factor of $100 \gamma$ has been assigned to this parameter and finally the fuzzy model becomes:

$$
\begin{aligned}
& S^{11}: \text { If }\left(x_{1} \text { is } M_{1}^{1}\right) \text { and }\left(x_{2} \text { is } M_{2}^{1}\right) \text { then } \\
& \dot{x}_{2}=0.1642+15.0164 x_{1}-0.3271 x_{2}-1.2458 u \\
& S^{12}: \text { If }\left(x_{1} \text { is } M_{1}^{1}\right) \text { and }\left(x_{2} \text { is } M_{2}^{2}\right) \text { then } \\
& \dot{x}_{2}=0.4848+14.6366 x_{1}+0.0002 x_{2}-1.1546 u \\
& S^{13}: \text { If }\left(x_{1} \text { is } M_{1}^{1}\right) \text { and }\left(x_{2} \text { is } M_{2}^{3}\right) \text { then } \\
& \dot{x}_{2}=0.1642+15.0162 x_{1}+0.3272 x_{2}-1.2458 u \\
& S^{21}: \text { If }\left(x_{1} \text { is } M_{1}^{2}\right) \text { and }\left(x_{2} \text { is } M_{2}^{1}\right) \text { then } \\
& \dot{x}_{2}=-0.0073+15.4272 x_{1}+0.0172 x_{2}-1.4291 u \\
& S^{22}: \text { If }\left(x_{1} \text { is } M_{1}^{2}\right) \text { and }\left(x_{2} \text { is } M_{2}^{2}\right) \text { then } \\
& \dot{x}_{2}=15.5778 x_{1}-0.0003 x_{2}-1.4536 u \\
& S^{23}: \text { If }\left(x_{1} \text { is } M_{1}^{2}\right) \text { and }\left(x_{2} \text { is } M_{2}^{3}\right) \text { then } \\
& \dot{x}_{2}=-0.0072+15.4287 x_{1}-0.0170 x_{2}-1.4291 u \\
& S^{31}: \text { If }\left(x_{1} \text { is } M_{1}^{3}\right) \text { and }\left(x_{2} \text { is } M_{2}^{1}\right) \text { then } \\
& \dot{x}_{2}=-0.0001+15.1478 x_{1}+0.3000 x_{2}-1.3232 u \\
& S^{32}: \text { If }\left(x_{1} \text { is } M_{1}^{3}\right) \text { and }\left(x_{2} \text { is } M_{2}^{2}\right) \text { then } \\
& \dot{x}_{2}=-0.2646+14.9965 x_{1}+0.0080 x_{2}-1.2568 u \\
& S^{33}: \text { If }\left(x_{1} \text { is } M_{1}^{3}\right) \text { and }\left(x_{2} \text { is } M_{2}^{3}\right) \text { then } \\
& \dot{x}_{2}=-0.0942+15.1516 x_{1}-0.2821 x_{2}-1.3232 u
\end{aligned}
$$

The mean square error is 0.0333 which represents the equilbrium point of the physical system, i.e., $a_{0}^{22}=0$. It can be clearly noticed in this model that the distance between adjacent subsystems is relatively small. It can also be noticed that the distance between the parameters of various adjacent control rules is relatively small. This in turn, strengths the hypothesis of designing a local control algorithm for each subsystem.

As observed, the results obtained through the parameter weighting method are always better than with the original T-S method. In fact, the proposed method correspond to the best possible result using TS method when the interval covering tends to totality, but this limit is not achievable since the problem would no longer be solvable.

\subsection{Control of an inverted pendulum using the proposed FLC-VSC}

The control of the inverted pendulum is a widely used performance measure of a controller, since this system is unstable and highly nonlinear. The objective is to maintain the inverted pendulum upright with $\theta$ despite small disturbances due to wind or system noises. The aim is to move the pendulum to its instable equilibrium position, i.e., $x_{1}=x_{2}=u=0$.

In this section, the proposed FLC-VSC is applied to stabilize and eliminate the chattering in the inverted 


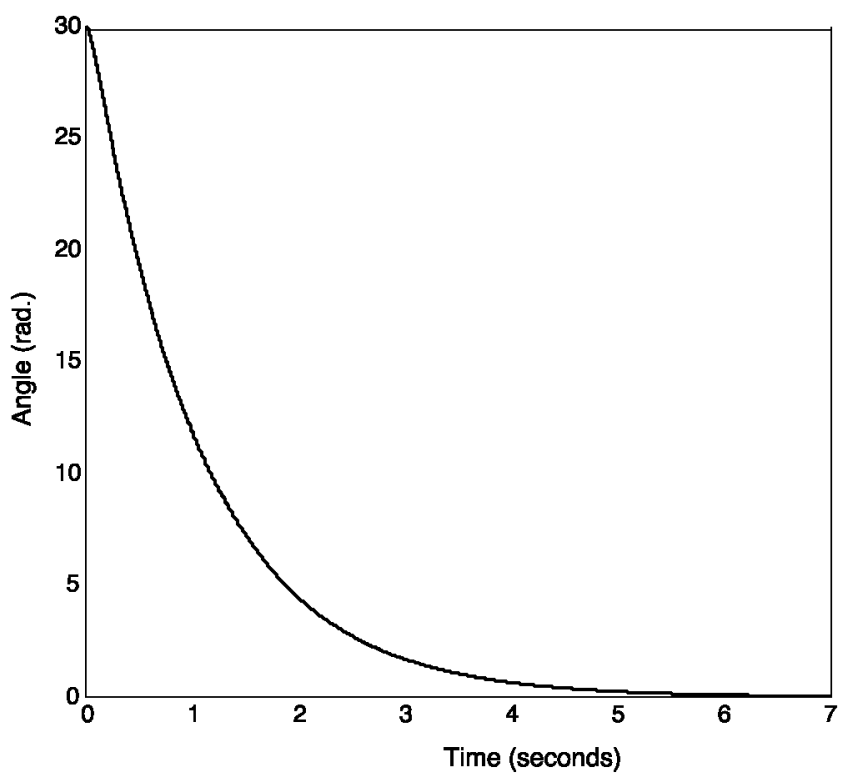

Fig. 8. Transient response of the inverted pendulum subjected to an initial condition.

pendulum estimated by the parameter weighting method. Supposing that the desired switching function is:

$$
s(x)=\left[\begin{array}{ll}
1 & 1
\end{array}\right] x(t)
$$

The boundary layer is 0.5 and $\mathrm{K}=10$. The proposed FLC-VSC becomes:

$$
\begin{gathered}
R^{(11-)}: \text { If } x_{1} \text { is } M_{1}^{1} \text { and } x_{2} \text { is } M_{2}^{1} \text { and } s \text { is } M^{-} \\
\text {then } u=-7.4609+11.4367 x_{1}+0.5285 x_{2} \\
R^{(110)}: \text { If } x_{1} \text { is } M_{1}^{1} \text { and } x_{2} \text { is } M_{2}^{1} \text { and } s \text { is } M^{0} \\
\text { then } u=0.0966+11.4367 x_{1}+0.5285 x_{2} \\
R^{(11+)}: \text { If } x_{1} \text { is } M_{1}^{1} \text { and } x_{2} \text { is } M_{2}^{1} \text { and } s \text { is } M^{+} \\
\text {then } u=7.6541+11.4367 x_{1}+0.5285 x_{2} \\
\vdots \\
R^{(33-)}: \text { If } x_{1} \text { is } M_{1}^{3} \text { and } x_{2} \text { is } M_{2}^{3} \text { and } s \text { is } M^{-} \\
\text {then } u=-7.5061+11.2244 x_{1}+0.5155 x_{2} \\
R^{(330)}: \text { If } x_{1} \text { is } M_{1}^{3} \text { and } x_{2} \text { is } M_{2}^{3} \text { and } s \text { is } M^{0} \\
\text { then } u=-0.0851+11.2244 x_{1}+0.5155 x_{2} \\
R^{(33+)}: \text { If } x_{1} \text { is } M_{1}^{3} \text { and } x_{2} \text { is } M_{2}^{3} \text { and } s \text { is } M^{+} \\
\text {then } u=7.3359+11.2244 x_{1}+0.5155 x_{2}
\end{gathered}
$$

Fig. 8 shows the transient response of the inverted pendulum controlled by the proposed FLC-VSC controller subjected to an initial condition of $30^{\circ}$. The results obtained show that the system is stabilized by applying the proposed FLC-VSC. Fig. 9 shows the robustness of the proposed controller subjected to disturbances of $5^{\circ},-3^{\circ}$ and $9^{\circ}$ respectively. Fig. 10 shows several trajectories of the system for several initial conditions.

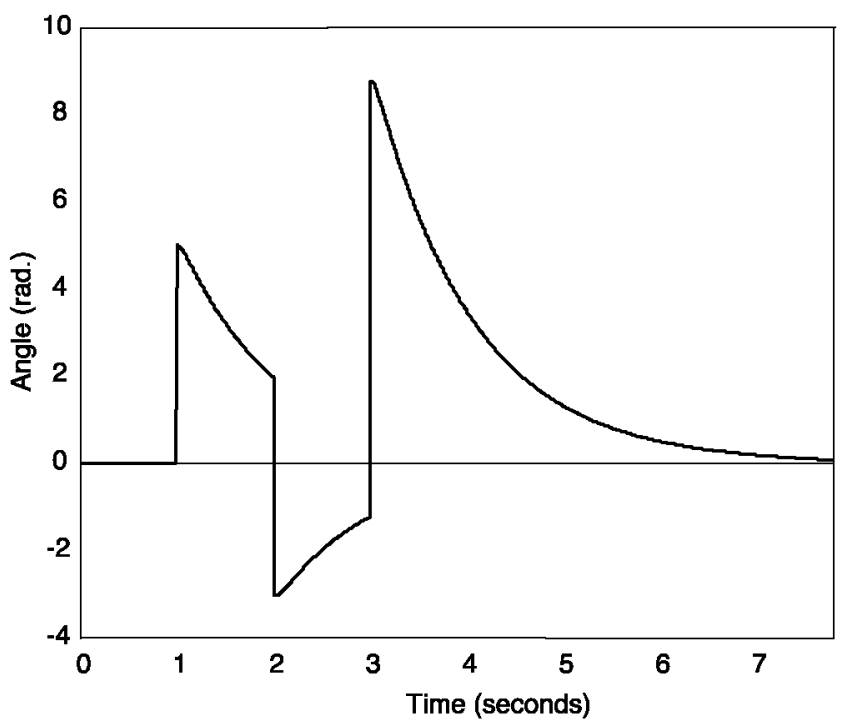

Fig. 9. Transient response of the inverted pendulum subjected to disturbances.

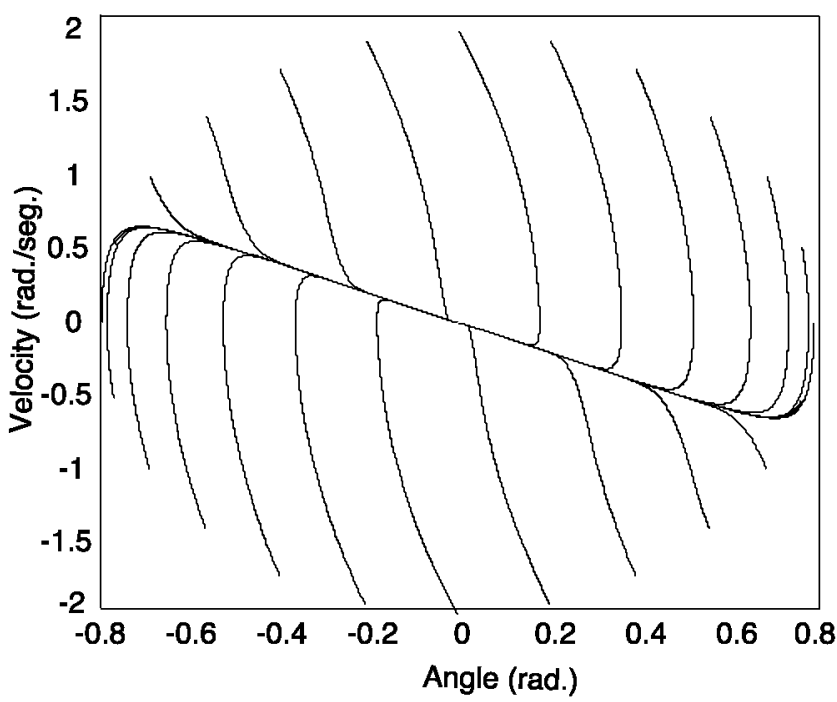

Fig. 10. Trajectories of the states of the system for several initial conditions.

\section{CONCLUSION}

A design of a FLC-VSC with new functions for chattering reduction without sacrifycing invariant properties are proposed. The main feature of the proposed method is that the switching function is added as an additional fuzzy variable and will be introduced in the premise part of the fuzzy rules together with the state variables.

The robustness of any control design depends mainly on the modelling accuracy of the controlled system. The main problem encountered in modelling \#a T-S model is that it can not be applied when the MFs are overlapped by pairs. 
An estimation approach has been developed to improve the local and global approximation and modelling capability of T-S identification methodology. The parameter weighting method has been proposed to reduce the error between the original system and the identified one. We show that parameter tuning of the weighting method is an effective method in the optimization of T-S fuzzy model. This variety of proposals for approximating T-S model can be seen as an advantage so that its parameters can be approximated to a linear model in an equilibrium point, which gives these parameters a physical sense and allows that their values become close to other parameters in adjacent rules. Thus, the similarity and closeness between the parameters in adjacent rules have the great advantage of ignoring the modeling error.

It should be noted that the systems modeled by applying the T-S model are smooth without large variations between adjacent subsystems. Moreover, the identification with the methodology of parameters tuning penalizes the distance to the initial parameters of the system. We can then conclude that the modeling error is very small with respect to the control effect, therefore it can be considered negligible and thus we can design a controller for each subsystem. This result can be generalized in a similar manner to any system of any dimension. It can also be noted that the distance between the parameters of various adjacent control rules is relatively small. This in turn, strengths the hypothesis of designing a local control algorithm for each subsystem.

An inverted pendulum mounted on a cart is chosen to evaluate the robustness, effectiveness and remarkable performance of proposed approach and the high accuracy obtained in approximating nonlinear systems in comparison with the original T-S model. Simulation results indicate the potential, simplicity and generality of the algorithm. In this paper, we prove that these algorithms converge very fast, thereby making them very practical to use. The application of the proposed FLC-VSC shows that both alleviation of chattering and robust performance are achieved.

\section{REFERENCES}

1. Al-Hadithi, Basil M., A. Jiménez, and F. Matía, "New Methods for the Estimation of T-S Model Based Extended Kalman Filter and its Applications to Optimal Control for Nonlinear Systems", Optim. Control, Appl. Methods, Published online in Wiley InterScience (www.interscience.wiley.com). DOI: 10.1002/oca.

2. Baranyi, P., "SVD-based reduction to MISO TS models", IEEE Trans. Ind. Electron., Vol. 51, No. 1, pp. 232-242 (Feb. 2003).
3. Baranyi, P., P. Patton, R. J. Korondi, and H Hashimoto, "Trade-off between approximation accuracy and complexity for T-S fuzzy models", Asian J. of Control, Vol. 6, No. 1, pp. 21-33 (March 2004).

4. Baranyi, P., P. Korondi, R. J. Patton, and H Hashimoto, "Global Asymptotic Stabilisation of the Prototypical Aeroelastic Wing Section via TP Model Transfromation”, Asian J. Control, Vol. 7, No. 2, pp. 99-111 (2005)

5. Baranyi, P., L. T. Kóczy, and T. D. Gedeon, "A Generalized Concept for Fuzzy Rule Interpolation”, IEEE Trans. Fuzzy Syst., Vol. 12, No. 6, pp. 820-837 (Dec. 2004).

6. Cao, S. G., N. W. Rees, and G. Feng, "Analysis and design for a class of complex control systems- Part I: Fuzzy modeling and identification", Automatica, Vol. 33, pp. 1017-1028 (1997)

7. Cavallo, A.,'High-order fuzzy sliding manifold control.", Fuzzy Sets Systems, 156: 249-266 (2005).

8. Chen, J. Y., "Rule regulation of fuzzy sliding mode controller design: Direct adaptive approach", Fuzzy Sets Syst, Vol. 120, pp. 159-168 (2001).

9. Gang, F.,'A survey on analysis and design of modelbased fuzzy control systems", IEEE Trans. Fuzzy Syst., Vol. 14, No. 5, pp. 676-697 (Oct. 2006).

10. Hseng,T., S. Li, and S.-H., Tsai, "Fuzzy Bilinear Model and Fuzzy Controller Design for a Class of Nonlinear Systems”, IEEE Trans. Fuzzy Syst, Vol. 15, No. 3, pp. 494-506 (Jan. 2007).

11. Ha, Q. P., Q. H. Nguyen, D. C. Rye, and H. F. DurrantWhyte, "Fuzzy sliding mode controllers with applications", IEEE Trans. Ind. Electron, Vol. 48, No. 1, pp. 538-46 (Jan. 2001).

12. Hua, C. and G. D. Xinping Guan, "Variable structure adaptive fuzzy control for a class of nonlinear time-delay systems", Fuzzy Sets Syst., Vol. 148, pp. 453-468 (2004).

13. Huang, S.-J. and W.-C. Lin, "Adaptive fuzzy controller with sliding surface for vehicle suspension control", IEEE Trans. on Fuzzy Syst., Vol. 11, No. 4, pp. 550-559 (August, 2003)

14. Hwang, C.-L., "A novel takagi- sugeno-based robust adaptive fuzzy sliding-mode controller", IEEE Trans. on Fuzzy Syst., Vol. 12, No. 5, pp. 676-687 (Octuber, 2004).

15. Hwang, C.-L. and H.-Y. Lin, "A fuzzy decentralized variable structure tracking control with optimal and improved robustness designs: theory and applications", IEEE Trans. Fuzzy Syst., Vol. 12, No. 5, pp. 615-630 (Octuber, 2004).

16. Kung, C.-C. and T.-H. Chen, "Observer-based indirect adaptive fuzzy sliding mode control with state variable filters for unknown nonlinear dynamical systems", Fuzzy Sets Syst., Vol. 155, pp. 292-308 (2005).

17. Jacob, S. and J. Munighan, "Designing fuzzy controllers from a variable structure standpoint", IEEE Trans. 
Fuzzy Syst., Vol. 5, No. 1, pp. 138-144 (February 1997).

18. Jiménez, A., B. M. Al-Hadithi, and F. Matía, "An Optimal T-S Model for the Estimation and Identification of Nonlinear Functions", WSEAS Transactions on Systems and Control, Vol. 3, No. 10, pp. 897-906 (October, 2008).

19. Jiménez, A., B. M. A1-Hadithi, and F. Matía, "Improvement of Takagi-Sugeno Fuzzy Model for the Estimation of Nonlinear Functions", Asian Journal of Control, Published online in Wiley Online Library (wileyonlinelibrary.com). DOI: 10.1002/asjc.310, Vol. 14, No. 2 (2012).

20. Johansen, T. A. , R. Shorten, and R. Murray-Smith, "On the interpretation and identification of dynamic TakagiSugeno models", IEEE Trans. Fuzzy Syst., Vol. 8, No. 3, pp. 297-313 (Jun. 2000).

21. Kim, J., Y. Suga, and S. Won, "A New Approach to Fuzzy Modeling of Nonlinear Dynamic Systems With Noise: Relevance Vector Learning Mechanism", IEEE Trans. Fuzzy Syst, Vol. 14, No. 2, pp. 222-231 (Apr. 2006).

22. Kóczy, L. T. and K. Hirota, "Size Reduction by Interpolation in Fuzzy Rule Bases", IEEE Trans. Syst., Man, Cybern. Part-B, Vol. 27, No. 1, pp. 14-25 (Feb. 1997).

23. Kumar, M., R. Stoll, and N. Stoll, "A Min-Max Approach to Fuzzy Clustering, Estimation, and Identification", IEEE Trans. Fuzzy Syst., Vol. 14, No. 2, pp. 248-262 (Apr. 2006).

24. Lam, H. K. and F. H. Leung, "Fuzzy rule-based combination of linear and switching state-feedback controllers", Fuzzy Sets Syst., Vol. 156, pp. 153-184 (2005).

25. Liang, C.-Y. and J.-P. Su, "A new approach to the design of a fuzzy sliding mode controller”, Fuzzy Sets Syst., Vol. 139, pp. 111-124 (2003).

26. Lo, J.-C. and Y.-H. Kuo, "Decoupled fuzzy sliding-mode control”, IEEE Trans. Fuzzy Syst., Vol. 6, No. 3, pp. 426-435 (August 1998).

27. Matía, F., B. M. A1-Hadithi, A. Jiménez, and P. S. Segundo, "An affine fuzzy model with local and global interpretation”, Appl. Soft Comput., Vol. 11, pp. 4226 4235 (2011).

28. Simon, D., "Design and rule base reduction of a fuzzy filter for the estimation of motor currents", Int. J. of Approx. Reasoning, Vol. 25, pp. 145-167 (2000).

29. Simon, D., "Training Fuzzy Systems with the Extended Kalman Filter”, Fuzzy Sets Syst., Vol. 132, pp. 189-199 (2002)

30. Skrjanc, I., S. Blazic, and O. Agamennoni, "Interval Fuzzy Model identification Using 1 -Norm”, IEEE Trans. Fuzzy Syst., Vol. 13, No. 5, (Oct., 2005).
31. Szeidl, L. and P. Várlaki, "HOSVD Based Canonical Form for Polytopic Models of Dynamic Systems", Journal of Advanced Computational Intelligence and Intelligent Informatics, Vol. 13. No. 1. 2009, pp $52-60$.

32. Takagi, T. and M. Sugeno, "Fuzzy identification of systems and its applications to modeling and control", IEEE Trans. Syst., Man Cybern., Vol. SMC-15, No. 1, pp. 116-132 (Jan. 1985)

33. Taur, J. S., C. W. Tao, and L. MChan, "Adaptive fuzzy terminal sliding mode controller for linear systems with mismatched time-varying uncertainties", IEEE Trans. Syst., Man Cybern. Part B Cybern., Vol. 34, No. 1, pp. 255-262 (Feb. 2004).

34. Tao, C. and J., Taur, "Design of Fuzzy Controllers with Adaptive Rule Insertion", IEEE Trans. Syst., Man Cybern. Part B Cybern., Vol. 29, pp. 389-397 (1999).

35. Tikk, D., P. Baranyi, and R. J. Patton, "Polytopic and TS Models are Nowere Dense in the Approximation Model Space", Proc. IEEE Int. Conf. Syst. Man Cybern. (SMC'02), Hammamet, Tunisia, Proc. on CD, (2002).

36. Tikk, D. and P. Baranyi, "Comprehensive Analysis of a New Fuzzy Rule Interpolation Method", IEEE Trans. Fuzzy Syst., Vol. 8, No. 3, pp. 281-296 (Jun. 2000).

37. Tikk, D., P. Baranyi, and Patton, R. J., "Approximation properties of TP forms and its consequences to TPDC design framework", Asian J. of Control, Vol. 9, No. 3, pp. 221-231 (September 2007).

38. Tong, S. and H.-X. Li, "A fuzzy adaptive sliding-mode control for mimo nonlinear systems", IEEE Trans. Fuzzy Systems, Vol. 11, No. 3, pp. 354-360 (June 2003).

39. Utkin, V. A., "Sliding modes and their applications in variable structure systems", Moscow: Mir, 1978, in English (1978).

40. Yam, Y. and L. T. Kóczy, "Representing membership functions as points in high dimensional spaces for fuzzy interpolation and extrapolation", IEEE Trans. Fuzzy Syst., Vol. 8, No. 6, pp. 761-772 (Dec. 2000).

41. Yam, Y., M. L. Wong, and P. Baranyi, "Interpolation With Function Space Representation of Membership Functions", IEEE Trans. Fuzzy Syst., Vol. 14, No. 3, pp. 398 411 (Jun. 2006)

42. Ying, H., "General SISO Takagi-Sugeno fuzzy systems with linear rule consequent are universal approximators," IEEE Trans. Fuzzy Syst., Vol. 6, No. 4, pp. 582-587 (Nov. 1998)

43. Zeng, K., N. Y. Zhang, and W. L. Xu, "A comparative study on sufficient conditions for Takagi-Sugeno fuzzy systems as universal approximators", IEEE Trans. Fuzzy Syst., Vol. 8, No. 6, pp. 773-780 (Dec. 2000). 